\title{
LXXXIV. Telescopic vision
}

\section{G. Johnstone Stoney M.A. Sc.D. F.R.S.}

To cite this article: G. Johnstone Stoney M.A. Sc.D. F.R.S. (1908) LXXXIV. Telescopic vision , Philosophical Magazine Series 6, 16:96, 950-979, DOI: 10.1080/14786441208636575

To link to this article: http://dx.doi.org/10.1080/14786441208636575

$$
\text { 册 Published online: } 21 \text { Apr } 2009 .
$$

Submit your article to this journal

Џ Article views: 2

Q View related articles ¿ 


\section{$[950]$}

LXXXIV. Telescopic Vision.

By G. Johnstone Stoney, M.A., Sc.D., F.R.S.*

\section{Part III.-How the Image is Formed, and of what PARTIALS IT CONSISTS.}

\section{[Plate XII.]}

Explanation of terms :-

1. u f W, undulation of flat waves, like the light of wave-length $\lambda$ received from a star.

2. u s W, undulation of spherical waves, like the light of wavelength $\lambda$ received from a luminous point.

3. Stellades and Punctades-see $\$ \S 62$ and 69 . Observation-A $u f W$ is a special kind of $u s W$; and a stellade is a special kind of punctade.

4. Complete image and its partials-see $\$ \$ 56$ and 80 .

5. Spile and anti-spile-see Chapter 3 , in the November Phil. Mag. p. 796.

\section{CHAP'TER 5.}

Outline of the Argument.

54. WWE have now to enter upon the most important part of our undertaking, in which we are to find out why it is that when a large and a small object exactly similar to one another are examined with the same telescope, the large one will be seen satisfactorily, while the small one, though of precisely the same shape, will if small enough appear when viewed through the telescope to be transformed into something unlike itself. In the last chapter we have met with specimens of such distorted appearances and we have now to learn the cause of these imperfections, and of other imperfections due to similar causes.

Hitherto in examining these objects (see Chap. 4) we have employed diffused light as the light to illuminate object $z^{\prime}$, in order that by doing so we may reproduce in our experimental apparatus the same optical conditions as those under which natural objects are seen through telescopes, see $\S 80$. But this mode of illumination will no longer suffice, because so long as we allow the light incident upon $z^{l}$ to be diffused light the whole phenomenon of the formation of its image in the pro-telescope is too complicated for investigation. It will be shown that what then happens is due to the simultaneous operation of a vast number of optical causes which produce different effects, and which in producing these effects act independently of one another. What, accordingly, we have to do is to separate these causes, and study what each does and why it does it; and then,

$$
\text { * Communicated by the Author. }
$$


having ascertained this, we shall inquire what the final outcome will be when these causes, or any selected group of them, are allowed to come simultaneously into operation.

55. These independent causes are in fact the several $u f W$ 's (undulations of flat waves) into which the diffused light incident upon $z^{\prime}$ is resolvable and which we may call $\mathrm{U}_{1}, \mathrm{U}_{2}, \mathrm{U}_{3}$, \&c. A part of each of these U's passes through the opening at $z^{\prime}$ which represents in our experimental apparatus some supposed detail upon the planet which is being examined by the astronomer. The part of this $U$ which passes through $z^{\prime}$ becomes to the left of $z^{\prime}$ what is called a 'beam' of light. This beam is of the kind which light from a star produces when allowed to pass through an opening in a shutter. We shall analyse this beam and find it to be a definite and complex optical phenomenon. We learn much about the uf W's (or u s W's) into which it may be resolved and of which it therefore consists, by introducing a contrivance (lens $\mathrm{L}^{\prime}$ ) by which the light of each ufW (or u s W, as the case may be, see $\$ 68$ ) will be concentrated into a speck of light upon plane $\mathrm{Y}^{\prime}$, where the simultaneous presence of the specks from all the uf $W$ 's of which the beam consists will constitute what we call the Concentration Image produced by that beam.

56. The light of the beam after becoming this concentration image upon plane $\mathrm{Y}^{\prime}$, continues its progress towards the left, and if it or a part of it is admitted into the protelescope $\mathrm{T}^{\prime}$, it will produce at $x^{\prime}$ within the pro-telescope that image of object $z^{\prime}$ which the light admitted to the pro-telescope is capable of forming. This is the image which the pro-telescope can furnish, when the incident light which renders object $z^{\prime}$ visible has been the light of the particular U which we bave allowed to illuminate that object. Let us suppose that it is $U_{1}$ which we have so employed, and let us call the image of $z^{\prime}$, which is then formed at $x^{\prime}$, $\mathrm{P}_{1}$. Similarly when $\mathrm{U}_{2}$ is the incident light, the image of $z^{\prime}$ will be $\mathrm{P}_{2}$, and so on. These P's we may call the partials, or partial images of $z^{\prime}$, which the U's when acting separately can produce. When the light incident upon $z^{\prime}$ was diffused light, all the U's were brought into operation, and the image then formed at $x^{\prime}$, which we may call the complete image, was due to the simultaneous presence there of all the partials, and may therefore be represented by the expression $\mathrm{P}_{1}+\mathrm{P}_{2}+\mathrm{P}_{3}+\& \mathrm{c}$. The complete image therefore results from light being superposed upon light at $x^{\prime}$. Now when light is superposed upon light, one or other of two things happens. If the lights that are superposed can permanently 
interfere with one another, it is an interference effect which will result from their being both present; but if they are lights which are incapable of permanent interference, their being made to co-operate upon disk $x^{\prime}$ only causes an illumination at each point of that disk which is the sum of the illuminations which they would have separately produced at that point. As we shall presently find, it is with this second and simpler case that we have here to deal. And accordingly what we have to do is to determine what the partial images $\mathrm{P}_{1} \mathrm{P}_{2}$ \&c. are, and then to add the illuminations which they separately produce at each point of disk $x^{\prime}$, in order to learn what is the brightness at that situation in the complete image at $x^{\prime}, i . e$. in the image which is identical with what the astronomer can under the most favourable conditions see in his telescope.

\section{CHAPTER 6.}

\section{Analysis of diffused light incident upon a small object.}

The task with which we have to grapple is to find ont by what process these images are really formed, for it requires but little consideration to satisfy ourselves that the explanation by Geometrical Optics, and other explanations which have been offered are either illusory or insufficient.

57. Geometrical Optics is a body of deductions from the supposition that light consists of rays-a supposition which is hypothesis not theory. A theory means a supposition which wo think may be true: a hypothesis is a supposition we expect to be useful. The only thing to be seen to when examining a theory, is whether it is correct or incorrect; for the merit of a theory is simply to be true, irrespectively of whether man can make much or little, or indeed any, use of it. On the other hand a hypothesis is a supposition of which the express intention is that it shall be helpful, and which is legitimate if it enables us to make any real progress in our work. No doubt a hypotbesis may also be a theoryin other words, a supposition which we entertain in the expectation that it will assist us in our investigation may also be the supposition which we think correctly describes what is going on in nature, but it by no means needs to be so: and in fact the best, i.e. the most useful, hypotheses are often of the kind that make no pretence to being true. The correct objective theory of light appears to be that light consists objectively of waves of alternating electro-magnetic stresses advancing through the æther; whereas the whole of 
Geometrical Optics, which is one of our most useful sciences, is built upon the supposition that light consists of rays-a supposition which it would be an error to mistake for a theory of light, that is for an attempt to define what light really is. That which makes the supposition deserving of our acceptance is that it is eminently useful and therefore legitimate as hypothesis. In Geometrical Optics what we investigate is the succession of events, not in nature, but in a model of nature. We substitute in this model machivery more easily handled than any which operates in nature, every step in the progress of which can be foretold by the application of singularly easy mathematical analysis, can be represented by easily understood diagrams, and can be imagined and followed without difficulty by students who possess but little skill. The justification of this bypothesis is that it is so easily handled, and that it yields results which are true within ascertainable limits. It can be shown that the hypothesis that light consists of rays furnishes correctly positions which are in close relation with the situations really occupied by the several parts of an optical image, although what it offers as the image differs in material respects from any real image. 'Thus 'the Geometrical Image,' as it is called, presents us with an unlimited amount of detail, most of which must be regarded as false because it is detail which does not exist in the images produced by nature. The hypothesis is very useful within definable linits, but may seriously mislead if it is misapplied. Accordingly, when we seek to ascertain what the image really is, we must abandon Geometrical Opties and have recourse to an investigation based on what we are entitled to accept as theory.

58. If we place at $z^{\prime}$ in our experimental apparatus (see Plate XII.) a small object representing some feature or features upon the planet, and if we illuminate it by diffused light, we shall see in the pro-telescope at $x^{\prime}$ an image of that object.

The diffused light which is to be incident upon $z^{\prime}$ may be light which has issued from an image of the sun as in \$ 39 (see p. 801 of last month's Phil. Mag.), or, when we do not need it to be of great intensity, it is often more convenient to provide it by a suitably placed piece of illuminated white paper or by light reflected from a cloud. Whatever is the source of the light it will be convenient to call it $\sigma$; and what we have now to ascertain is the succession of events by which a part of the light emitted by $\sigma$ succeeds in becoming an image of $z^{\prime}$ at $s^{\prime}$ in the pro-telescope. 
59. This sequence of events naturally divides itself into three stages- $1^{\circ}$, From $\sigma$, the source of light, to the object $z^{\prime} ; 2^{\circ}$, From the object to the pro-telescope; and $3^{\circ}$, Within the pro-telescope-in correspondence with whch our study of the events will have to consist of three inquiries: $1^{\circ}$, How the light emitted by $\sigma$ and incident upon $z^{\prime}$ is to be analysed? $2^{\circ}$, What happens to the portion of this light which gets past $z^{\prime}$, upon its journey between plane $\mathrm{Z}^{\prime}$ and plane $\mathrm{Y}^{\prime}$, which latter stands in front of the pro-telescope? And $3^{\circ}$, By what further behaviour does this light or some part of it afterwards form the image at $x^{\prime}$ ? In the present Chapter we shall deal with the first of these inquiries, reserving the second and third for consideration afterwards.

60. To fix our ideas we may suppose the source of light $\sigma$ to be a bright round disk of paper two inches in diameter placed at the situation of $s^{\prime}$ in Plate XII., i.e. at a distance of about a metre and a half to the right of object $z^{\prime}$; and we may conceive of this disk as though it were divided into patches $d \sigma_{1}, d \sigma_{2}, \& c .$, hexagonal like a honeycomb, and of sufficiently small size (which they will be if about $\frac{1}{3} \mathrm{~mm}$. across). Then the light of wave-length $\lambda$ emitted from these small $d \sigma$ 's and reaching $z^{l}$ will, under the theorem proved in $\$ 29$ (see p. 335 of the Phil. Mag. for last August), be equivalent to $\mathrm{ufW}$ 's (undulations of flat waves) travelling towards $z^{\prime}$, which will be of the same number as the patches. inasmuch as one will advance from each $d \sigma$ in the direction of the line from the middle of that patch to the middle of $z^{\prime}$ together with an exceedingly small amount of $\mathbf{r}$ (residual light) due to the rulings formed on plane $Z^{\prime}$ by the $P$ 's and $-Q$ 's described in $\$ 29$. These rulings produce scarcely any illumination at the part of plane $\mathrm{Z}^{\prime}$ where $z^{\prime}$ is situated. In fact, if the $d \sigma$ 's are as small as we have described them and if the object $z^{\prime}$ is of sufficiently moderate size, this residual light will be so faint that in making experiments it may be quite left out of account, and the whole of the light of wave-length $\lambda$ reaching $z^{\prime}$ may be regarded as exclusively consisting of the same number of uf $W^{\prime}$ 's as there are $d \sigma$ 's in $\sigma$, one arriving at $z^{\prime}$ from each of the $d \sigma^{\prime}$ s.

61 . Hitherto we have considered only light of one wavelength, whereas the light emitted by $\sigma$ being white light contains light of all the wave-lengths included within the visible part of the spectrum. Accordingly, the light transmitted from each one of the $d \sigma^{\prime}$ s to $z^{\prime}$, is to be regarded as consisting of uf W's of all these wave-lengths, all travelling in the same direction, viz., in the direction of the line from the middle of the $d \sigma$ to the middle of object $z^{\prime}$. These from 
the mathematician's point of view will be infinite in number, since he requires to be able to push matters to their limit, in order to comply with the requirements of mathematical analysis; but when our object is either to interpret experiments, or to deal with nature, the whole range of wave-lengths may be divided into groups, $d \lambda_{1}, d \lambda_{2}$, \&c., each of a sufficiently small range, and then, as explained in $\S 31$, a single $u f W$ may be substituted for each of these groups. When this has been done the light incident upon $z^{\prime}$ from any one of the $d \sigma$ 's will consist of a large but finite number of uf W's, of different wave-lengths all travelling in one direction. Let $m$ be the number of these 'groups,' i.e. of the $d \lambda$ 's, witbin the visible part of the spectrum, and let $n$ be the number of $d \sigma^{\text {'s }}$ in disk $\sigma$, then will the entire of the visible light incident upon $z^{\prime}$ consist of $m n$ u f $W^{\prime}$ s, of which $m$ of the uf W's of different wave-lengths will have issued from each $d \sigma$-together with traces of $\mathrm{r} I$ (residual light), too faint to need to be taken into account.

62. The visible light which inhabitants upon the earth receive from a white star is of the same kind as that received by the $z^{\prime}$ of our experimental apparatus from any one of the $d \sigma$ 's of the source of light. They both consist of the same u f W's, of different wave-lengths, with in addition a tiny amount of residual light, which is too faint to need to be considered. It will be convenient to have a name for this kind of light, and it will in the present paper be called a stellade; which will therefore mean light of the same kind as we receive from a star. The statement at the end of the last paragraph may now be put into the following more convenient form :-

If $\sigma$, the source of light, is divided into $n$ sufficiently small patches, $d \sigma_{1}, d \sigma_{2}, \& \mathrm{c}$., and if the wave-lengths present within the visible part of the spectrum are divided into $m$ sufficiently small groups, $d \lambda_{1}, d \lambda_{2}, \& c$., then will the visible light which is thrown by disk $\sigma$ upon object $z^{\prime}$, consist of $n$ stellades, on $\theta$ from each $d \sigma$, advancing along the $n$ directions from the centres of the $d \sigma$ 's to the centre of $z^{\prime \prime}$; and each of these stellades will consist of $m \mathbf{u} f W^{\prime}$ 's, of the wave-lengths that correspond to the middles of the small groups, $d \lambda_{1}, d \lambda_{2}$, \&c.together with the residual light referred to in $\S 29$, which is so very faint that it may legitimately be disregarded.

This resolution of the light incident upon $z^{\prime}$ into $n$ stellades, and of each stellade into $m \mathrm{ufW}$ 's, answers the first of the three inquiries proposed in $\$ 59$, viz.: How is the light in its first stage, i.e between $\sigma$ and $z^{\prime}$, to be analysed?

63. It should be borne in mind that the uf $W$ 's into which 
we have here resolved the incident light are of the complex type referred to in $\$ 31$ (August no. of the Phil. Mag.p. 337), that is to say, they are uf W's the waves of which need not be alike except as regards wave-length, but in which, throughout the whole extent of each wave, there will be no difference between one part of that wave and another part of the same wave. Restrictions seem to limit the differences that actually prevail between the successive waves on any one $\mathrm{ufW}$, consequent upon events that go on within the superficial molecules of visible matter. This is a subject one part of which is discussed in a paper in Vol. IV. of the Scientific Transactions of the Royal Dublin Society (1891), p. 563, from which discussion it appears that the light emitted by a visible body is due to the activities of the negative electrons within its molecules, and that the motion of any one electron, however intricate its path, is resolvable into elliptic partials each of which will emit light of a definite wave-length *.

\section{CHAPTER 7. \\ Analysis of a beam of light.}

When we intend that the light incident upon object $z^{\prime}$ in our experimental apparatus (see Plate XII.) shall be diffused light, we employ a source of light which we have called $\sigma$. A part of this light will ultimately become the image of $z^{\prime}$ which may then be seen in the pro-telescope ' $\mathrm{T}^{\prime}$ at $x^{\prime}$; and in order to trace how this comes to pass we have divided the advance of the light from $\sigma$ to $x^{\prime}$ into three stages.

64. The first stoge of this progress, which is from $\sigma$ to $z^{\prime}$, we have studied in the last chapter, and have found that if $\boldsymbol{\sigma}$ be divided into $n$ sufficiently small patches $d \sigma_{1}, d \sigma_{2}, \& c$, each of these will transmit towards object $z^{\prime}$ a single stellade of light (light of the kind we receive from stars), and that each of these stellades will consist of $m$ u f W's (undulations of flat waves), one for each of the $m$ wave-lengths of which we have found that white light may legitimately be regarded as consisting-along with a residuum of light obeying a different law of propagation, which however at the middle of object $z^{\prime}$ and for a certain distance round that point is so

* We have some reason to suspect that the more definite motions of negative electrons are accompanied by surgings of the associated positive electricity. It may be to these less definite displacements of positive electricity that we are to look for the explanation of subsidiary effects which have been observed, even possibly including anomalous dispersion. 
faint that its presence may be disregarded unless object $z^{\prime}$ is too large. We shall be careful not to make it too large; and may therefore regard the light incident upon $z^{\prime}$ as consisting of $n$ stellades and each stellade of $m u \mathrm{f}$ W's, these stellades advancing upon $z^{\prime}$ in the $n$ directions represented by lines from the middles of the $\bar{d} \sigma^{\prime}$ s to the middle of $z^{\prime}$.

65 . Let us now select some one of these stellades and study what further happens to it. To do this we must be able to deal with it separately ; that is we must free it from the presence of the other stellades which will accompany it. so long as $\sigma$ is the source of light. This is easily done by removing $\sigma$ from the experimental apparatus and employing $s^{\prime}$, the pro-star, as the source of light. The pro-star will then illuminate $z^{t}$ with a single stellade, and this may legitimately take the place of the stellade whose future history we want to trace, if we make its light fall upon $z^{\prime}$ in the right direction. This we can do by availing ourselves of the adjustments provided at the $B$ end of the board BE. See $\$ 22, p .333$.

66. What we have in the present Chapter to study are the events that will then occur between object $z^{\prime}$ and the plane $\mathrm{Y}^{\prime}$ which stands in front of the pro-telescope. To do this satisfactorily, we must confine our attention to light transmitted to the astronomical telescope from some definite object upon the planet, and the object we shall select as the first to be dealt with is an object like the planet Neptune, and in some degree like the Solis Lacus upon Mars, at longitude $87^{\circ}$ and south latitude $27^{\circ}$ upon the maps of the so-called ' canals upon Mars.' The Solis Lacus is a small but conspicuous spot upon the image of the planet, of a shape not far from round, and of a diameter on the planet which when the planet comes nearest to us subtends at the distance of the earth an angle not far from our standard angle $\epsilon$. We are not at present concerned with the faint traces of detail which are supposed to have been at times detected upon its image, since the question to which we are at present seeking an answer is, what appearance the outline of an object of some such shape and size will have in the astronomical telescope, if such an object exists upon the planet? The object we shall suppose will, for simplicity, be an object exactly round, and subtending exactly angle $\varepsilon$ at the distance of the astronomical telescope from the planet. The object which we have assumed to exist upon the planet will be adequately represented in the experimental apparatus by a round hole in copper-foil at $z^{\prime}$, illuminated by light coming from beyond it. This will present in the pro-telescope the appearance of a bright object with dark surroundings, whereas the Solis 
Lacus is seen as a dark object with brighter surroundings; but this difference is immaterial, since the optical conditions for seeing a bright object upon a dark background, and a dark object of the same shape and size upon a bright background, are known to be the same.

67. The object on the planet which we have chosen for our first experiment being a round object of such a size that it subtends angle $\epsilon\left(2^{\prime \prime} \cdot 062648\right)$ at the distance of the earth will be adequately represented in the OEA (observatory experimental apparatus) at $z^{\prime}$, by a round hole $1 \mathrm{~mm}$. in diameter, in the screen of copper-foil $\mathrm{Z}^{\prime}$, when the carpenter's square which supports the screen has been pushed into its place near the right-hand surface of lens $L^{\prime}$, with $z^{\prime}$ opposite to the middle of the lens. This is to be illuminated by the single stellade of light issuing from pro-star $\boldsymbol{s}^{\prime}$, and the observer can bring the direction in which this stellade is incident upon object $z^{\prime}$ into any of the directions along which light from the various parts of a source of diffused light, such as $\sigma$, would reach that object. When the incident light reaches screen $Z^{\prime}$, most of the light is stopped by that screen, the only light which is allowed to advance beyond the screen being that which passes through the round opening $z^{\prime}$. We need not consider what happens to the rest of the light as we are concerned only with what is called the 'beam of light' which advances past the hole.

68. We have to find out what this beam of light is. It is plainly no longer the simple stellade which encountered screen $Z^{\prime}$, and which consisted of u $\mathrm{f} W$ 's of different wave-lengths all travelling the same way. But it is light of some kind, and as such may be resolved into u f w's or in any other way that is legitimate. One of these legitimate resolutions (and the one which will most assist us when experimenting with an apparatus in which lens $\mathrm{L}^{\prime}$ has a focal length less than the whole of the distance from that lens to plane $\mathrm{Y}^{\prime}$ ) is a resolution into usw's (undulations of spherical wavelets) which shall radiate from the several points of a surface $W$ (see Plate XII.), of such a form and so situated that its points are the conjugate foci with reference to lens $L^{\prime}$ of the several points of plane $\mathrm{Y}^{\prime}$ which stands in front of pro-telescope ' $\mathrm{T}^{\prime}$. The positions of these conjugate foci may be determined by Geometrical Optics, since this is a case in which Geometrical Optics leads to a correct result. Furthermore, instead of employing all the points of surface $W$ as the centres of innumerable undulations of infinitesimal intensity, we may, as in other similar cases, distribute a large but finite number of points over surface $W$ at sufficiently short intervals and 
regard these as the centres of u s W's (undulations of spherical waves) each of which will take the place of a sheat of the u s w's and may be bright enough to be seen. It is accordingly into these u $\mathrm{s}$ W's that, when dealing with experiments, we shall resolve the light between object $z^{\prime}$ and lens $L^{\prime}$.

69 . Let $p_{1}, p_{2}, \& c$. be the numerous but not innumerable points which we have supposed to be distributed cver surface $W$, and which are the centres of the spherical undulations into which we have resolved the light within the limited space between screen $Z^{\prime}$ and lens $L^{\prime}$. As this light contains light of all the wave-lengths present in white light, there will, in general, be spherical undulations of some or all of these wave-lengths emanating from each of the $p$ 's. Any complete system of mndulations of the different wave-lengths with which we are concerned, and all of them consisting of spherical waves which have one common centre we shall find it convenient to call a punctade of light, which will then mean light of the same kind as that which issues from a luminous punstum or optical point, whether white or coloured. Accordingly we may regard the light within the limited space between screen $Z^{\prime}$ and lens $L^{\prime}$ as resolved into punctades $\mathrm{P}_{1} \mathrm{P}_{2}$, \&c., which have their centres at the points $p_{1} p_{2}$, \&c.; and each of these punctades will consist of the us W's of different wave-lengths into which we may resolve white or coloured light emanating from a point.

70. Having got so far, we shall facilitate the next step of our inquiry by substituting instead of the real source of light $s^{\prime}$, and the obstruction it meets with at screen $Z^{\prime}$, the much simpler machinery which we can imagine by making the hypothesis that this source of light and that obstruction have been removed, and that at the same time the puncta $p_{1} p_{2}$, \&c. upon surface $W$ have been rendered luminous and are now what emit the light which exists between plane $Z^{\prime}$ and lens $L^{\prime}$. This is a hypothesis of the same legitimate kind as the familiar one which we make when we find it convenient to treat light reflected from a plane mirror as having come directly from the virtual images of the objects in front of the mirror instead of indirectly from those objects themselves.

71. When we view matters in this way we see that these punctades are incident upon lens $\mathrm{I}^{\prime}$, and accordingly that lens $L^{\prime}$ will concentrate the light of any one of them, suppose of $\mathrm{P}_{1}$, into an image of the kind which that lens is capable of forming of the luminous punctum from which we have asked the reader to regard the light of this punctade as having issued. This will be into an image which we may call $Q_{1}$, formed upon a small patch of plane $\mathrm{Y}^{\prime}$, which we shall call 
$q_{1}$, and of which the middle point will be the point upon that plane which is conjugate to point $p_{1}$ upon surface $W$. How small the patch is which will be occupied by this image, may be seen from the following considerations. The image of a point formed by a lens is of the same kind as the image which we have called a star-burst. This image was investigated by Airy and consists of a central boss of bright light (sometimes called the spurious disk of the star) surrounded by relatively faint coloured rings, of which, usually, only a few of the inner ones can be seen. A very large percentage of the light is concentrated into the central bright boss and nearly the whole into it and the two inner appendage rings. Now, from Airy's formulæ we find that in the author's apparatus the patch occupied by the central boss and its two inner appendages is less than a tenth of a millimetre in diameter, and that the central boss in which most of the light is concentrated is only a few hundredths of a mm. in diameter *. We are therefore justified when making experiments in regarding the light of each of the u s W's incident upon lens $\mathrm{L}^{\prime}$, as being concentrated into an image small enough to be regarded as a speck of light upon plane $\mathrm{Y}^{\prime}$. In the author's apparatus there are about 100 of these specks in every square mm. of plane $\mathrm{Y}^{\prime}$; and, of course, the size of these specks can be made as much smaller as the observer chooses by substituting for $L^{\prime}$ a lens of correspondingly larger aperture.

72. Accordingly, what the observer will see on looking through his Steinheil lens at B will be the image which we have called the concentration image upon plane $Y^{\prime}$. This he may regard as consisting of the specks of light which occupy the patches $q_{1}, q_{2}$, \&c. into which plane $\mathrm{Y}^{\prime}$ has been divided. He should then reflect that each one of these specis, as we may call them, is the concentrated light of one of a definite set of punctades of light into which the beam of light which has advanced to the left of screen $Z^{\prime}$ has been able to resolve itself; and that what he sees on plane $\mathrm{Y}^{\prime}$ supplies the following information about that resolution. In the first place the position of the speck upon plane $\mathrm{Y}^{\prime}$-let us suppose that it is $q_{1}$-can be made to indicate to him the direction in which

* The image in the author's apparatus is in fact larger, because of the large spherical aberration of telescope objective $L^{\prime}$ when employed to form an image of a near object. This might have been corrected by using as lens $L^{\prime}$, two telescope objectives put back to back, but this more elaborate arrangement was not adopted, as the simpler arrangement was found to work well in practice. 
the component travelled, which has been concentrated into that speck*

In the next place, the intensity at the various situations of the concentration image tells the observer the relative intensities of the several components of the beam which are concentrated into the corresponding specks.

And, thirdly, the colours of the concentration image at those situations indicate in some degree the various proportions in which wave-lengths are mixed in the components, whether they be punctades or stellades. This very considerable amount of information is increased when the observer adds to it what he may infer from the circumstance that all this light has been furnished by the single stellade emitted from pro-star $s^{\prime}$, which is the light that illuminates object $z^{\prime}$. This light reached $z^{\prime}$ in the same phase and in the same state of polarization throughout each wave-front. Accordingly, all the light which advanced beyond screen $z^{\prime}$, and with which alone we are concerned, started from $z^{\prime}$ in the same phase and in the same state of polarization, and accordingly if at any subsequent stage the different parts of this light are brought together again after having spent equal times in their several journeys from $z^{\prime}$, they will arrive at that destination (as, for instance, at $x^{\prime}$ in the pro-telescope) under such conditions that they can produce there the kind of persistent interference effects of which human eyes can take cognizance. This we shall find a very important circumstance: upon it depends the competence of this light to form at $x^{\prime}$ the image which it displays there.

73. An object upon the planet which is small enough to subtend not more than a few seconds of angle at the distance of the earth may have its outline correctly represented by the boundarv of a mere hole of the proper shape and size at $z^{\prime}$. And the experiments that can then be made with it will

* The direction in which that component of the beam travelled across whatever free space was left for it to the left of sereen $Z^{\prime}$, is made known to the observer by the position upon plane $Y^{\prime}$ of the speck of light into which the light of that component is concentrated. He has only to picture to himself the ray (of Geometrical Optics) from that speck to the optical centre of lens $L^{\prime}$, with its continuation to the right of lens $L^{\prime}$. The portions of this ray which are in air are parallel to one another, and their direction when reversed iudicates the direction in which the component in question was travelling until it encountered lens L'; that is to say, this is the direction in which the stellade advanced, if the resolution of the beam is regarded as being into stellades, and it is the direction of the central radius of the punctade, if the resolution is into punctades. Either of these resolutions is legitimate.

Phil. Ma.g. S. 6. Vol. 16. No. 96. Dec. 1908. 
show what approach the astronomical telescope can make to reproducing this outline in its image of the planet. If the astronomical telescope shows further detail upon the image of the small object, the conditions under which this further detail becomes visible must be made the subject of a separate investigation. What a simple hole at $z^{\prime}$ suffices to do is to investigate the vision which can be obtained of the outline, and this is usually what is wanted in practice. In the few cases when more is required we must substitute something other than a mere hole at $z^{\prime}$. Howerer, for the present we confine our attention to mere holes, and to those of elementary shapes such as round, rectangular, or triangular. For forms less simple much the best course is to inspect, and if necessary measure, the concentration image in the $O E$ apparatus, which will then give the astronomer the information he requires.

74. A circhlar opening is the first with which we shall deal, inasmuch as with a small hole of this shape in screen $Z^{\prime}$ it is specially easy to foresee what the optical effect on plane $Y^{\prime}$ will be, when light from the pro-star $s^{\prime}$ is admitted through that opening. For, in the first place, since object $z^{\prime}$ is small the light which reaches it from the pro-star is not appreciably distinguishable from the light which would reach it from a luminous point on surface $W$, and accordingly we may regard the light which reaches $z^{\prime}$ as light that has emanated from that luminous point, which we may call $w$. Again, objective $L^{\prime}$ and the Steinheil lens at $B$ may be regarded as the objective and eyepiece of a telescope with which the observer is looking at luminous point $w$. The aperture of his telescope is limited by the size of the round hole $z^{\prime}$, which we shall suppose cuts it down to being one millimetre. It thus appears that what the observer sees through his Steinheil lens is the image of luminous point $w$ which the telescope just described has been able to form when its aperture is only $1 \mathrm{~mm}$. Now the image of a luminous point in a telescope is of the same kind as the image of a star. It is the kind of image which we have called a star-burst, all the principal details of which are known to us from Airy's investigation, and the dimensions of which can be computed by his formulæ. The image consists of a very bright central boss of nearly white light, often spoken of as the spurious disk of the star, surrounded by coloured rings which are so much fainter and so rapidly deteriorate in brightness, that Airy's formulæ only give full particulars for the central boss and the two inner rings which are nearly all that can be seen of the phenomenon in astro- 
nomical telescopes. The sizes of these depends upon the wave-length of the light, and therefore differs for the different colours of the spectrum. With light of one wave-length everything would be definite. At the centre the intensity is a maximum, and at a certain distance from the centre it falls to cipher. This distance will be the radius of a circle of no intensity, which will separate the central boss from the first ring; and similar circles of no intensity separate the successive luminous rings from one another. By slightly simplifying Airy's formulæ (see Transactions, Cambridge Philosophical Society, vol. v. 1835, p. 287), the angular radii of the innermost three of these, as seen from the optical centre of lens $L^{\prime}$, are found to be

$$
\theta_{1}=(1 \cdot 22) \frac{\lambda}{\mathrm{A}}, \theta_{2}=(2 \cdot 27) \frac{\lambda}{\mathrm{A}}, \theta_{3}=(3 \cdot 24) \frac{\lambda}{\mathrm{A}}, .
$$

where $\theta_{1} \theta_{2}$ and $\theta_{3}$ are the angular radii as seen from the optical centre of the objective, $\lambda$ is the wave-length of the light for which the computation is made, and $A$ is the aperture of the telescope, which in the case supposed is $1 \mathrm{~mm}$. Hence, on plane $\mathrm{Y}^{\prime}$ which is about a metre from the objective, the radii of these innermost dark circles will approximately be

$$
\left.\begin{array}{l}
t_{1}=1000(1 \cdot 22) \lambda \\
t_{2}=1000(2 \cdot 27) \lambda \\
t_{3}=1000(3 \cdot 24) \lambda
\end{array}\right\}, . . . \quad . \quad .
$$

and if we insert 0.7 of a micron for $\lambda$, which is about the longest wave-length of the conspicuous parts of the spectrum, we find that for that wave-length the radii of the three dark circles will be

$$
t_{1}=0.854 \mathrm{~mm} ., t_{2}=1.589 \mathrm{~mm} ., t_{3}=2 \cdot 268 \mathrm{~mm} \text {. }
$$

These the observer sees magnified seven times by his Steinheil

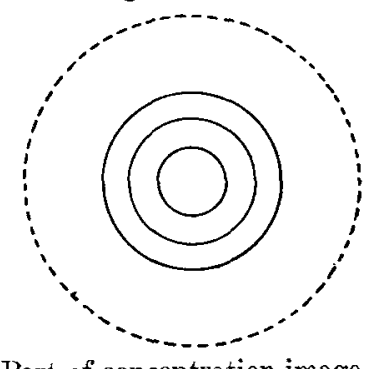

Part of concentration image.

lens. But in the annexed figure they are magnified only four times, as this magnification will make the diagrams in 3 R 2 
this paper of conven :ent size. When white light is used the central boss will occupy the space within the inner circle, and the innermost two appendage rings will occupy the spaces between the circles, since the overlapping of spectra does not become conspicuous till farther out. If the phenomenon is particularly well seen three or four more of the luminous rings will also be visible. The space within the dotted line of the figure would include five of the appendage rings, which are usually all that we need consider. If one wishes to see what the more definite eflect is with monochromatic light, the absorbing contrivance known as a thallium screen may be held before the eye.

75. By these considerations we learn that when the opening at $z^{\prime}$ is round, and when the light incident upon $z^{\prime}$ has been a single stellade of light, its concentration image will be identical except in size with the image of a star as seen in a telescope. It is what we have called a star-burst, and with a round opening is the kind of star-burst which Airy investigated. If the opening at $z^{\prime}$ is of some other shape, the concentration image on plane $\mathrm{Y}^{\prime}$ produced when $z^{\prime}$ is illuminated by a single stellade will still be a star-burst or image of a star, for in fact it will be the kind of image which a telescope would furnish if its objective had this new shape. Several such images produced by stars were examined by Sir John Herschel, and some of them are figured in Plates IX. and X. of his article on Light in the Encyclopcedia Metropolitana. But they are displayed in fuller detail by the $\mathrm{OE}$ apparatius, and with great additions to what can be made visible by examining the images of stars in telescopes. The astronomer when using the $\mathrm{OE}$ apparatus should bear in mind that in all cases the concentration image produced by a single stellade of incident light must be essentially a star-burst or image of a star whatever the shape of the opening at $z^{\prime}$ may be. It is the image which the telescope would furnish if its objective were of the shape of the opening or openings which he has made at $z^{\prime}$. And he will tind a study of the concentration image which will result from different forms of openings or from rows of openings most suggestive. When the form of the opening is polygonal the spectra which are associated in its concentration image with a central boss of light are no longer circular as they are when $z^{\prime}$ is a round hole. They become detached patches of coloured light, and some of the more distant ones are more conspicuous than the corresponding spectra when $z^{\prime}$ is round. This is a very instructive fact. It must be remembered that it is the same light which 
produces the concentration image, or some part of this light, which afterwards develops the image of object $z^{\prime}$ which is seen it $x^{\prime}$ in the pro-telescope; and if $z^{\prime}$ is of an angular shape, the light of the more distant spectra must be admitted to the pro-telescope in order that the vertices in the angular image may be seen sharply defined. If the aperture of the pro-telescope is unable to admit the light of these distant spectra, the angles in the image of $z^{\prime}$ will appear blunt and not reaching out as far as they should. This seems to be the case with most if not all of the polygonal spiles in the image of Mars which are bounded by the dark streaks in the image which have been called canals. And this is a state of things which must apparently cause dark specks to present themselves at the angles, and may be the true account of the dark specks upon Mars which have been interpreted as representing oases upon the planet.

76. Before closing this chapter it may be well to refer to a property common to all concentration images, which the reader is requested to keep constantly in view when investigating the process by which the light emitted by objects at $z^{\prime}$ forms images at $x^{\prime}$ in the pro-telescope. This property is that objects at $z^{\prime}$ which are similur but of different sizes will give rise to concentration images on plane $\mathrm{Y}^{\prime}$ which will also be similar, but of linear dimensions which will be inversely as the linear dimensions of the objects. See fig. 1, $\S 83($ p. 969).

The concentration image has a double meaning. It as it were looks backwards and analyses for us the beam of light which has passed through the opening at $z^{\prime}$; and it looks forward and furnishes the information about the image in the telescope which will occupy our attention in the next chapter.

\section{CHAP'TER 8.}

\section{How a Telescopic Image is formed.}

78. By combining what we have learned in the last two chapters, we obtain a complete reply to the second of the three questions propounded in $\$ 59$, in which we are asked how a beam of light is to be analysed. If, for example, the beam has come from the sun through a hole in a shutter, which opening may be of any form, we are first to conceive that a sheet of star-like bodies, sufficiently close together and sufficiently bright, take the place of the sun as the source of light. In Chapter 6 we found that the light reaching an opening of limited size in the shutter will be for practical 
purposes the same whether it comes from the sun or from this sheet of stars. Regarding it as coming from the stars, we at once see that the sunbeam may be regarded as an aggregate of star-beams, one coming through the opening in the shutter from each of the stars in the sheet of stars. The light which reaches the shutter from each star is the kind of light we have called a stellade; and lights from any two of these stellades, having come from independent sources, are lights incapable of producing with one another visible interference effects. One consequence of this is that the intensity of the sunbeam at any situation will be the mere sum of the intensities which the starbeams would separately produce at that place.

Knowing that light of any kind and therefore that a starbeam is capable of resolving itself into components which may be either stellades o: punctades, we learn in Chapter 7 much about these components. To gain this knowledge the observer inspects that concentration image which by the help of lens $\mathrm{L}^{\prime}$ will be produced upon plane $\mathrm{Y}^{\prime}$ whenerer light from pro-star $s^{\prime}$ passes through an opening at $z^{\prime}$ and becomes a star-beam. Knowing that each luminous speck of this concentration image is the concentrated light of one of the components of the star-beam, he is told by the position of the speck upon plane $\mathrm{Y}^{\prime}$ in what direction that component travelled between screen $Z^{\prime}$ and lens $L^{\prime}$; and from the intensity and colour of the speck he learns what the intensity and colour of the component are. When to this information we add that all parts of the light of this concentration image have started from $z^{\prime}$ in the same phase and in the same state of polarization at each instant of time, but that the light of any two of the concentration images may have been in various other phases and in rarious other states of polarization-when these particulars are added we have all the information we shall need in the inquiry upon which we are now to enter.

79. Let light from the pro-star $s^{\prime}$ be allowed to fall on screen $\mathrm{Z}$ '. A portion of this light passes through the opening at $z^{\prime}$, and becomes a starbeam between screen $Z^{\prime}$ and lens $L^{\prime}$, and this starbeam by the intervention of lens $L^{\prime}$ produces the image on plane $\mathrm{Y}^{\prime}$ which we have called the concentration image. After forming this image the light continues its advance so that the pro-telescope can be placed in such a position that the whole or some part of the advancing light will enter the pro-telescope, and will then form at $x^{\prime}$ the image of object $z^{\prime}$ which the portion of the light which gains admission to the pro-telescope is able to yield. 
80. The image formed from light that originally came from a star or pro-star we shall call a partial image, or partial. The partial image cannot be the same as the image of the corresponding feature on the planet which would be presented to the observer in his astronomical telescope. This is because the planet is an opaque body, the superficial molecules of which have been rendered luminous by light which has reached them from in front, and which bas set up such activities in their superficial molecules as scatter light in all forward directions. To represent this correctly in our experimental apparatus the light reaching $z^{\prime}$ from behind must be light that advances in all forward directions, or in other words it must be diffused light. And the image of $z^{\prime}$ which is then formed, and which is identical with that formed by the corresponding feature on the planet, is entitled to be spoken of as the complete image of $z^{\prime}$. To form this complete image in our apparatus it would suffice to illuminate $\tilde{z}^{\prime}$ from behind by light coming from a sheet of pro-stars instead of from a single one. This makes it obvious that the complete image is that image which is formed when all the partials formed by the individual pro-stars are simultaneously present on disk $x^{\prime}$. These partials, however, we can in the experimental apparatus study one by one. As they arise from light coming from different pro-stars, viz. from $d \sigma_{1}$, $d \sigma_{2}$, \&c., they come from independent sources of light, and therefore when they are all thrown down simultaneously upon disk $x^{\prime}$, the portions of light which they individually contribute and which form images at $x^{\prime}$ are unable to produce further interference effects than those which they separately produce. Hence we arrive at the important conclusion that when we have studied what the partials are when examined one by one, we can arrive at the brightness at any point of the 'complete' image, by simply adding together the illuminations which will be produced at that point by the partials when they are made to present themselves in succession.

81. In order to see the general outcome of this state of things, we shall examine how the principles that have been laid down will work out when applied to a special instance. The feature to be seen we shall suppose to be the simple boundary of the whole planet, when the planet is $1^{\circ}$, Neptune in opposition, and $2^{\circ}$, Mars when nearest to the earth in 1909. In other words the objects are nearly circular disks, and these we shall represent by circular openings of the proper sizes, at $z^{\prime}$, respecting which we are to discover of what partials their images in the pro-telescope will be built up 
when the pro-telescope is successively made to correspond to astronomical telescopes with apertures of 40,24, and 12 metric inches, i.e. with apertures of 100,60 , and 30 centimetres.

82. The angular diameter of Neptune in opposition is about $1 \frac{1}{3} \epsilon$, and the maximum diameter of Mars in 1909 will be $11 \cdot 655 \mathrm{\epsilon}$. We shall first deal with these magnitudes in the most obvious way, which is also the way best fitted for computation; and we will afterwards indicate another way of dealing with them which is more convenient to the experimentalist. The first or more obvious course is to imagine round openings in copper foil with diameters $1 \frac{1}{3} \mathrm{~mm}$. and $11.655 \mathrm{~mm}$. to be placed successively at $\hat{z}^{\prime}$, and to illuminate these by a single stellade of light incident upon them from the right. They, and the round opening $1 \mathrm{~mm}$. in diameter with which we have been already experimenting, would any of them produce on plane $Y^{\prime}$ the same kind of concentration image. These, however, would be of different sizes, their linear dimensions being inversely as the linear dimensions of the openings at $z^{\prime}$ to which they are due. Hence we can deduce the sizes of the others from the dimensions given in $\$ 74$; where we found that the concentration image presented when $z^{\prime}$ is a round hole $1 \mathrm{~mm}$. in diameter furnishes the values:

$$
t_{1}=0.854 \mathrm{~mm} ., t_{2}=1.589 \mathrm{~mm} ., t_{3}=2.268 \mathrm{~mm} .,
$$

where $t_{1}, t_{2}$, and $t_{3}$ are the radii of dark circles which separate the central boss of light and the first three of the appendage rings which with the central boss form the whole of the concentration image produced by light of wave-length $\lambda=0.7$ of a micron. This wave-length and wave-lengths shorter than it are all that we need take into account, as this range includes all the brighter parts of the spectrum of white light. Accordingly the central boss and its two inner appendage rings will lie within a circle of which the radius is $2.268 \mathrm{~mm}$. Airy's formulæ do not enable us to calculate the radii of the limiting circles that lie farther out; but their position can be seen and measured in the experimental apparatus, and it thus appears that a circle with a radius of $4.2 \mathrm{~mm}$. would nearly include all of the fifth ring. Appendage rings that lie farther out become so faint that they need not be taken into account unless we are considering the vision of a much brighter object than a planet. We may therefore safely assume that the whole of the effective part of the concentration image is bounded by a circle $4.2 \mathrm{~mm}$. in radius. This radius we shall call $\mathrm{T}$. 
83. Accordingly, when $z^{\prime}$ is a hole $1 \mathrm{~mm}$. in diameter, the radius of the whole of the efficient part of its concentration image upon plane $\mathrm{Y}^{\prime}$ will be

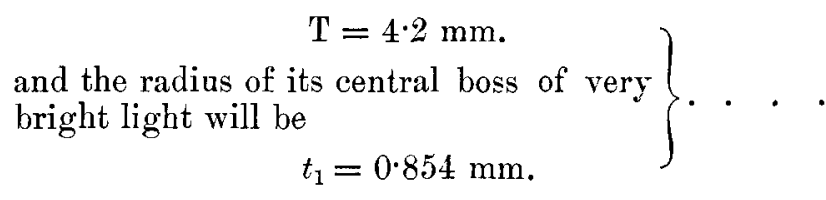

From these values we can compute what they would become if the diameter of the opening at $z^{\prime}$ were made successively $1 \frac{1}{3} \mathrm{~mm}$. and $11.655 \mathrm{~mm}$. so as to represent planets Neptune and Mars. We thus find that a round hole at $z^{\prime}$ which would represent Neptune in opposition, would make

$$
\left.\begin{array}{l}
4 \mathrm{~T}^{\prime}=12 \cdot 6 \mathrm{~mm} . \\
4 t_{1}^{\prime}=2 \cdot 562 \mathrm{~mm} .
\end{array}\right\}, \cdot . \quad \cdot .
$$

and that the hole at $z^{\prime}$ which would represent Mars when nearest to us in 1909 , i.e. on the 18 th of September, would make

$$
\left.\begin{array}{l}
4 \mathrm{~T}^{\prime \prime}=1.44 \mathrm{~mm} . \\
4 t_{1}^{\prime \prime}=0.293 \mathrm{~mm} .
\end{array}\right\} . \quad \cdot \quad . \quad . \quad .
$$

In writing these we have introduced a single dash when the equations refer to Neptune, and a double dash when they refer to Mars; and the radii have been multiplied by 4 because the annexed diagrams need to be on a larger scale than the scale on which the originals present themselves on plane $Y^{\prime}$. In these diagrams each inch upon the objective of the astronomical telescope is represented by a millimetre.

Fig. 1.

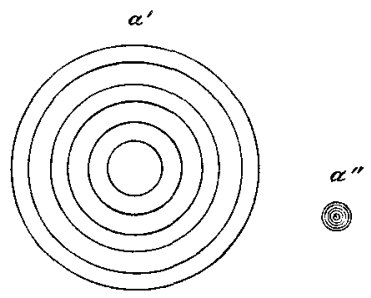

Concentration images of Neptune and Mars.

In fig. $1 a^{\prime}$ is four times the size of the concentration image upon plane $Y^{\prime}$ when $z^{\prime}$ represents Neptune, and $a^{\prime \prime}$ is four 
times what its size becomes when $z^{\prime}$ represents Mars. Fig. 2 will illustrate what kind of vision can be obtained of the outline of these planets if examined with a telescope of which

Fig. 2.

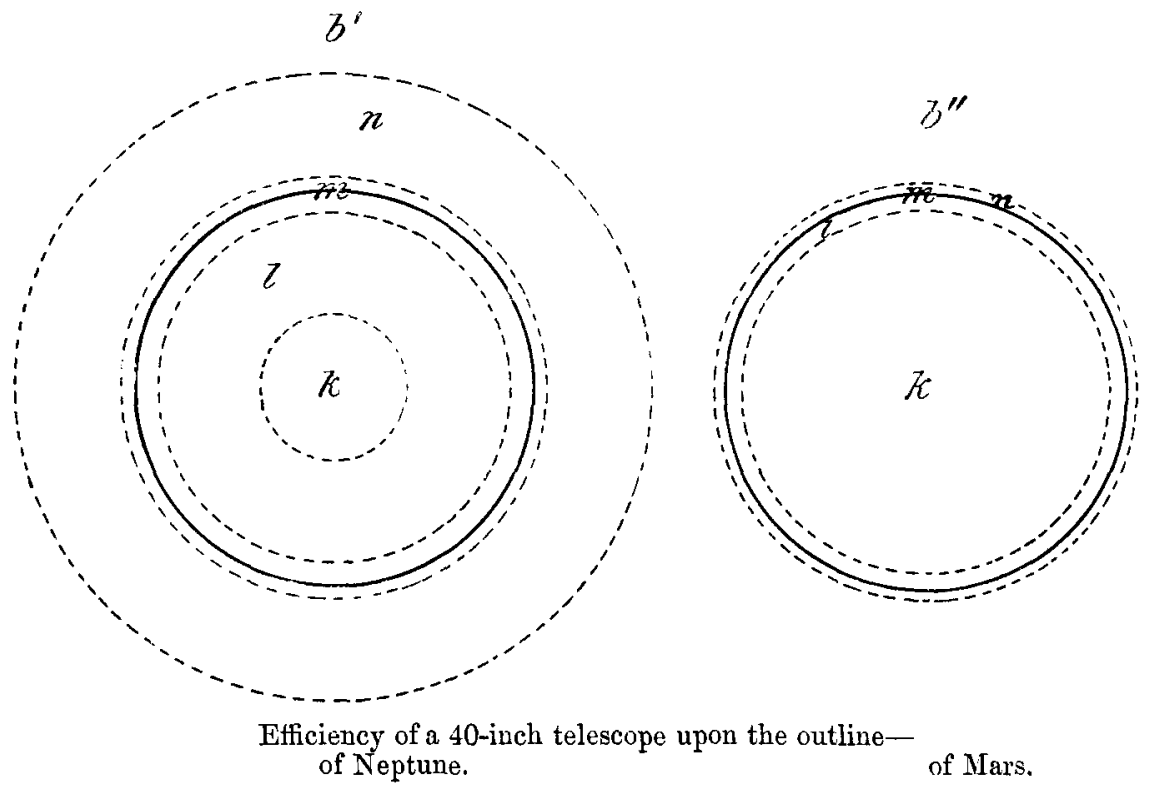

the aperture is a metre (i.e. 40 metric inches), and if the optical state of our atmosphere is sufficiently good, which it of course very rarely is. In this diagram the continuous line is the circumference of the objective, and the dotted circles separate it and the neighbouring space into four regions $k l m$ and $n$. The dotted lines nearest to the continuous line are at distances from it $=4 t_{1}^{\prime}$ in the left-hand figure, and $=4 t_{1}{ }^{\prime \prime}$ in the right-hand figure ; while the outermost and the innermost circles are distant from the outline of the objective, at distances from it $=4 \mathrm{~T}^{\prime}$ in the left-hand figure, and $=4 \mathrm{~T}^{\prime \prime}$ in the right-hand figure* .

* In the right-hand diagrams of figs. 2, 3, and 4, the distances of the four dotted circles from the continuous circle should be the lengths given for $4 \mathrm{~T}^{\prime \prime}$ and $4 t^{\prime \prime}$ in equation (7). Of these only the more distant dotted circles, at the distance $4 \mathrm{~T}^{\prime \prime}$ from the edge of the objective, can be represented in the diagrams. The other two, between which lies region $m$, can be imagined though too close to the continuous circle to be drawn. 
84. To make use of these diagrams we must remember that the 'complete image' in the pro-telescope is due to the simultaneous presence in that telescope of all the partials, and that a partial is produced whenever the whole or any part of the concentration image represented in fig. 1 is able to enter the pro-telescope. Now fig. $1, a^{\prime}$ is the concentration image formed by a stellade of light incident upon the proNeptune at $z^{\prime}$; and obviously if the direction of the incident stellade to which it is due, brings its middle point $o$ anywhere within space $k$, then the whole of the efficient light of that concentration image has got into the pro-telescope, and therefore the 'partial,' the image of $z^{\prime}$ which it will form, will be an image so good that we may practically regard it as an optimum partial. On the other hand, if othe centre of fig. 1 falls anywhere within space $l$, only part, although it will be the greater part, of the concentration image gains admission to the pro-telescope. The resulting 'partial' formed at $x^{\prime}$ will fall short of being the optimum, the defect being slight if $o$, the centre of the concentration image, reaches a part of region $l$ which is distant from the margin of the objective, while the defect goes on increasing the nearer that $o$ comes to the margin of the objective. This $l$ group of partials may be called fair images of $z^{\prime}$, and will some of them be almost good, others only mediocre.

The next stage is when $o$ the centre of the concentration image reaches space $m$. Under these circumstances part even of the light of its central boss is excluded from the protelescope and the resulting partial will be a decidedly bad image of $z^{\prime}$, and this defect becomes still worse when the centre of the concentration image lies farther from the objective within region $n$. If the centre of the concentration image lies farther out than region $n$, none of its light gets access to the pro-telescope and we are therefore not concerned with it. Now when the light incident upon $z^{\prime}$ is diffused light, as it must be when we want to represent correctly what occurs in nature, this light when analysed will furnish stellades that would send the centres of their concentration images to all the points represented in fig. 3, so that all the partials which have been described above are present upon disk $x^{\prime}$ and cooperate to produce there the 'complete image' of $z^{\prime}$. It is obvious that in the state of things represented in fig. 2, $b^{\prime}$, this complete image cannot be a very satisfactory one. It is to be regretted that when using a telescope we are unable to exclude any of the partials. It is convenient to divide them into the four groups which we call $k l m$ and $n$, depending upon the situations on plane $\mathrm{Y}^{\prime}$ (the plane of the 
figure) which the centres of their concentration images reach. The partials belonging to class $k$, if all the rest were excluded, would acting together form an exceedingly good image at $x^{\prime}$, since the partials of this group are all optimum partials. This is a state of things which the microscopist can realise by closing the iris-diaphragm under his condenser sufficiently to exclude the light which would develop any other partials than those of class $k$. This in using the microscope is called 'clearing the image.' We can do the same in the author's experimental apparatus, by calling into requisition an irisdiaphragm provided at $F$ (see Plate XII.). But the astronomer has no means of accomplishing it with his telescope. He must put up with all those mischievous bad partials, being present in the image which the telescope presents to him. And under the conditions represented in fig. $2, b^{\prime}$, they contribute so largely to the image that the resulting 'complete image' cannot yield a really good image of the outline of Neptune, which is what we want to see, although the astronomer would probably consider it quite a fair image. This is partly because of a very curious power which the experienced observer acquires of, in such cases, being able in some degree to detect the image which the better of the contributory partials would form, and being able to distinguish and exclude from his consideration some at least of the added defects.

By the movements provided at the $\mathrm{B}$ end of the board $\mathrm{BE}$, the observer can bring the concentration image successively into all the positions described in the last paragraph, and can then see for himself the partial images in the pro-telescope, which will result; and the astronomer would do well to familiarise himself with these details.

85. We have now to contrast with the above the image which the same telescope will furnish of an object of the same shape but of larger angular size, when the feature to be seen is the outline of the disk of planet Mars. Here when we distribute the partials into the four groups $k l m$ and $n$, we find this distribution represented by fig. $2, b^{\prime \prime}$. This diagram shows us that the optimum partials, which are those of group $k$, are greatly more numerous * than they were when Neptune was the object, and at the same time the partials of the three other groups, $l m$ and $n$, are much fewer. Hence the 'complete image' of the outline of the planet Mars which will result from their being all present,

* The numbers of the partials of each group are proportional to the areas allotted to them in figs. 2, 3 and 4 . The intensities of the partials have however to be taken into account as well as their number. 
is chiefly characterized by this great preponderance of optimum partials. The state of things which now prevails will furnish an image of the edge of the planet which any astronomer would pronounce to be an exceedingly sharp* image. Of course this will only happen, if the observation is made on one of those rare occasions when 'seeing' is very good.

86. We shall next consider how these same objects would appear in telescopes of less aperture, such as those with which the most successful observations have hitherto been made upon the images of Mars in telescopes. The apertures we will consider are apertures of 24 inches and of 12 inches. Proceeding as before, we find that the following figures indicate the proportions in which the four classes of partials will be present in these images when the objects under examination are the contours of Neptune and Mars.

Fig. 3.
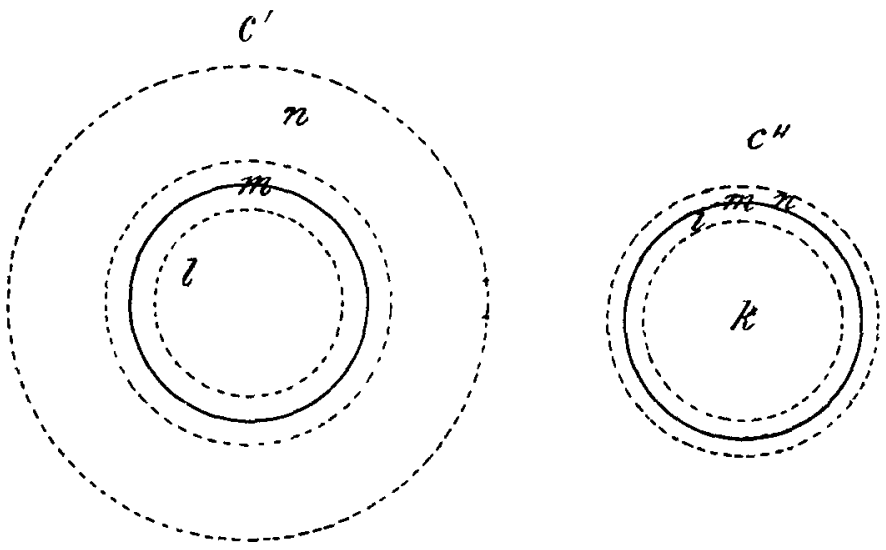

Efficiency of a 24-inch telescope upon the outlineof Neptune.

of Mars.

From fig. 3, $c^{\prime}$, we learn that when Neptune is examined with an aperture of 24 inches, the image will contain no partials of class k, i.e. none of the best kind; but that there will be present a considerable contingent of the partials of class $l$ (without however the best of that class). Those of class $l$ that are present would produce a fair image, were it not for the large admixture of partials of the other two

* This does not in the least imply that such vastly smaller details as the profiles of mountains upon the edge of the planet could approach to being made visible by a 40 -inch telescope. 
classes, $m$ and $n$, which are the kinds of partials which most tend to spoil an image. Owing to their presence the image of the edge of planet Neptune as seen in a 24-inch telescope cannot be assigned a higher place than that of a barely fair image of that feature of the planet.

87. The state of things will of course, become worse, if the aperture of the telescope is cut down to 12 inches. What will then happen is depicted in fig. $4 d^{\prime}$. From this diagram

Fig. 4.

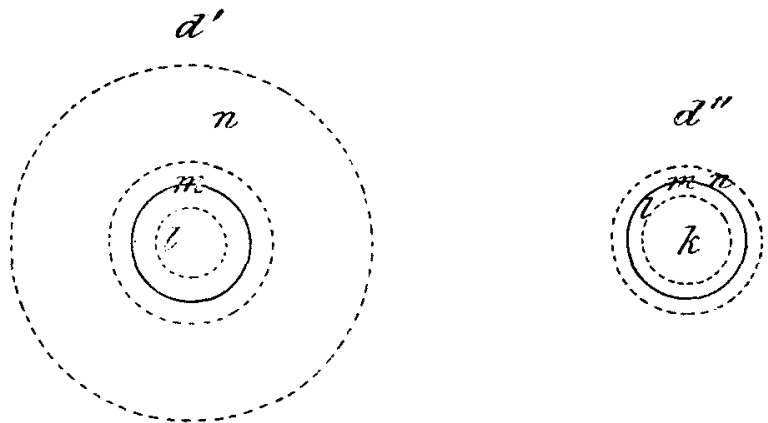

Efficiency of a 12-inch telescope upon the outlineof Neptune.

of Mars.

we see that the image of Neptune's outline is made up chiefly of partials of the two bad classes $m$ and $n$, superposed upon only a few of those of class $l$, and they only the less efficient of this class. The resulting 'complete image' will therefore poorly represent the real margin of the planet.

88. As before, we shall find a great contrast to the state of things described in the last few paragraphs when we turn the same telescopes upon planet Mars. The visions which they will afford of the outline of Mars are expressed diagrammitically in fig. $3 c^{\prime \prime}$ and fig. $4 d^{\prime \prime}$. From these we learn that even with the 12-inch telescope, the image of the contour of Mars when nearest the earth in 1909, will be decidedly well seen on a good night. The image will include a considerable number of the $k$ partials-the partials of the best kind-with not a very large admixture of partials of the three inferior kinds $l m$ and $n$. With the 24-inch telescope this satisfactory state of things will be improved on. The proportion in which the $k$ partials will now stand to the three other classes will have become a much greater proportion, with a corresponding improvement in the resulting " complete 
image' of the outline of the planet, which will be of the kind that an astronomer would pronounce to be excellent.

89. In order to show the efficiency of the proposed method of investigating telescopic vision, and of discovering the reason why it is encumbered with the imperfections which become troublesome when we endeavour to see minute objects, it has been thought that the best course was to describe how to apply the new method to some one class of objects; and the class chosen has been round or roundish objects such as are some of the spots on the image of Mars, including the polar caps at the times when these appear to be roundish or oval objects. The telescope is more successful in dealing with objects of this kind than with objects of angular or other irregular form. This is because in the latter case, as experiments with the OE apparatus will show, the concentration images which they develop upon plane $\mathrm{Y}^{\prime}$ do not, as with round objects, consist of a boss of white light surrounded by annular spectra alike in all directions round the boss and which after the first four or five become excessively faint, but consist of a central boss of some other form surrounded by spectra occupying detached patches upon plane $\mathrm{Y}^{\prime}$, of very unequal intensity in the various directions round the boss, and in some directions retaining sufficient brightness to need to be taken into account, when considerably farther from their central boss, than the fifth annular spectrum which is the most distant we have thought it necessary to retain when inquiring as to the vision of round objects. Long rows of exceptionally bright spectra are in such cases apt to radiate out in some directions from the central boss, and the more distant of these will be excluded if the aperture of the telescope is insufficient. This must have often happened when the 24-inch telescope and in a more intense degree when the 12-inch telescope have been employed to examine polygonal patches subtending at the telescope so small an angle, as do those brigher regions upon Mars which are enclosed by whatever on Mars correspond to the 'canals' upon the image, or by the darker 'seas.' When these outlying spectra are excluded from the telescope, one effect will be that the angles of the polygon will be rounded off, and such appearances as 'oases' and 'carets' will present themselves.

90. In all such cases the astronomer can make at $z^{\prime}$ a hole which will represent both in size and shape any supposed polygonal feature upon Mars. This is to be illuminated by the pro-star $s^{\prime}$, and he can then see through the Steinheil lens the concentration image it produces on plane $\mathrm{Y}^{\prime}$, and 
can measure the distance from the centre of the concentration image to the most distant appendage spectrum which ought to be retained. The distance so measured he is to employ as his $\mathrm{T}$ in constructing diagrams like those of figs. 2, 3, and 4, which will then reveal to him the kind of vision of the supposed object upon the planet which his astronomical telescope would be competent to supply.

91. Many experiments with the microscope throw additional light upon this interesting subject. One of these I may mention. The object was the proboscis of the Blowfly, and the part of the specimen which was made use of was

Fig. 5.

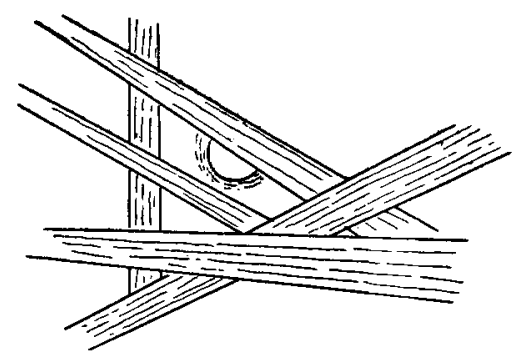

a small triangular patch of bright light which happened to be shut in between three of the hairs or bristles which grow near the base of the proboscis. These hairs had been pressed when mounting the specimen, so that they lay near to one another and nearly in a plane perpendicular to the optic axis. Another thinner hair which we may call the canal crossed the triangle and lay parallel to one side. This canal divided the triangle of light into a smaller triangle below, and a quadrilateral space above the canal. Within this quadrilateral was seen about balf of the base of another small hair, presenting the semicircular appearance in fig. 5, and being somewhat darker on the right-hand part of the semicircle than on the left. The rest of this hair lay outside the triangle of light to which attention is being called.

This object was examined through one of Zeiss's $24 \mathrm{~mm}$. apochromatics, over which an iris diaphragm bad been fitted to enable the observer to diminish its aperture to any desired extent. The succession of appearances while the aperture was being diminished was most suggestive. The object was fairly well seen until the aperture became rather small, but then on still further contracting the aperture a succession of new phenomena sprang into existence, until at a certain stage 
the appearance became that represented in fig. $6^{*}$, and was then utterly unlike the real object. The semicircle has

Fig. 6.

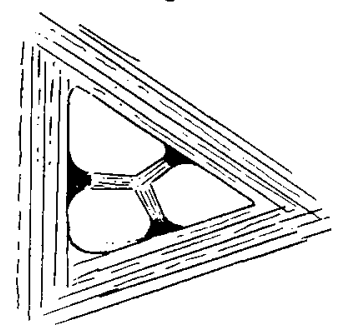

disappeared and instead of it and the one straight canal across the triangle we have what appear to be three canals of nearly equal thickness and abutting nearly perpendicularly upon the three sides of the entire triangular space. And at the same time 'carets' have developed themselves at the outer ends of these three optically produced canals. These misleading appearances, which sometimes metamorphose an object into something utterly unlike itself, are of the same kind as those by which the Astronomer who occupies himself upon minute details, is but too liable to be misled, unless he diligently avails himself of some such aids as those which the author has ventured in this memoir to recommend.

\section{NOTES.}

Nore 1. On control observations upon the Moon.- When in last month's Phil. Mag. (see p. 807), recommending the Astronomer diligently to make control observations upon the Moon when nearly full, the author omitted to point out that valuable observations of this kind can be made without having to provide the auxiliary telescope there suggested. With that telescope the exact optical conditions of the observations upon Mars can be reproduced, including the rision of Mars in the astronomical telescope, and of the Moon in the control apparatus, under equal magnification.

To make observations with the naked eye we have only to

* In looking at fig. 6 the reader is requested to exercise lis imagination, for none of the features as seen in the microscope had the hard outlines of our diagram of them. The three 'cauals' were dusky strealis with straight but nebulous edges, and the boundary of the triangular bright space was also nebulous. The 'carets' were the darkest parts of the image.

Phil. Mag. S. 6. Vol. 16. No. 96. Dec. 1908. 3 S 
provide round holes of the sizes given in column 4 of the table on p. 807. With a little care, these can be made with needles in softened copper foil (see $\$ 26, \mathrm{p} .331$ ). Of theso holes that one is to be selected which corresponds to the angular size of Mars at the time, which is given in the Nautical Almanac (see column 2 of the table). On looking with the naked eye through this hole at the full Moon, we see the Moon under the same optical conditions as when we view Mars through the astronomical telescope armed with a magnifying power equal to the number which is found in column 3 of the table. If we wish to have at our command an opportunity of using other powers, we must provide the small auxiliary telescope suggestod in $\S 48$.

Note 2. On the size of the Eulolon.-In the text (see \& 13) we have described the Eidolon, or object which appears to the observer to be what he is looking at, as of one special size. In this it was assumed that the observer had focussed his telescope so as to be able to scrutinise the eikon, the image presented to his eye, with most success. By adjusting the focus differently the eikon could be made to give the impression of a larger object at a greater distance; but it is best, as in the texr, to focus in the way that enables the olserver to scrutinise the image he sees to most advantage.

And, when focussing bis tolescope, the experienced observer will judge as to whether it is tocussed correctly by the vision he obtains of whatever is the most distinctly seen feature of the image presented to him. This in the case of "Mars will be the contour of the planet and not any of the objects upon its disk, and especially not any phenāko-spile.

Note 3. On lens $I^{\prime}$ of the $O E$ apparatus. - Since the focal length of lens $L^{\prime}$ is less than a metce, we have the concentration image affected by the spherieal aberration spoken of in the footnote on p. 960 . However, it is found that this defect does not sensibly impair the inage cren when the focal length of $L$ is as shopt as it is in the author's apparatus. Neither is it sensibly the worse because $s^{\prime}$ is a little further from $z^{\prime}$ than surface $W$, the conjugate of plane $Y^{\prime}$. If on the other hand the focal length were longer than a metre, the specks of the concentration image which are formed by each of the punctades (or stellades) into which the beam is analysed, would dilate and become less detinite as seen upon plane $\mathrm{Y}^{\prime}$, and if the focal length were excessive (which would have the same effect as removing lens $L^{\prime}$ from the apparatus) the diffuseness of its specks would impair our vision of the 
concentration image. This is what renders it necessary to retain lens $L^{\prime}$ as part of the apparatus. The concentration image upon plane $\mathrm{I}^{\prime}$ can be seen, but very poorly seen, without its intervention.

Of course, the experimental apparatus would most closely represent the Cosmical Apparatus described in Chapter 1, if the focal length of lens $\mathrm{L}^{\prime}$ were a metre, and if at the same time a collimating lens were introduced between $z^{\prime}$ and $s^{\prime}$, to parallelize the light from pro-star $\varepsilon^{\prime}$.

Note 4. On the analysis of light into $\mathrm{ufw}$ 's.-In all Mathematical and experimental investigations into Nature. what in each case we deal with is not the whole of th; activities that are going on, but some immense simplification of them effected by ignoring the bulk of what is really taking place, and considering only the small residues which are what can be perceived by man. Thus we can perceive a wind in air, which is in fact only a residual effect, a slight preponderance in one direction of all the momentums which are consequent upon the vastly swifter motions of the molecules between their enconnters; and these motions are themselves only the residues which remain orer as a preponderance in one direction of the enormously more subtile momentums that are going on within those travelling missiles.

So also, in his investigations of wave motion, the mathematician finds it necessary to substitute untextured mediums, devoid of all that majority of events which he ignores,--he has to substitute this simplification of nature for the real, immenstrably more complicated activities that are really in operation. The untextured medium is the mathematician's hypothesis ; and is legitimate because, under his handling of it, it furnishes results which are correct under certain detinable circumstances, though incorrect under others. They are correct whenever we have not to deal with distances that approach to being molecular. The mathematician, however, in order to carry out his work, has to explore the whole ficld, as he has to base liis reasoning upon limiting conditions which occur within the part of the field that, when he comes to interpret his results, is the very part that must be rejected. Resolutions into $\mathrm{u} f \mathrm{w}$ 's, - - undulations of wavelets -are of this kind, and are of ralue because although the results to which they lead at close quarters are incorrect, all the results to which they lead beyond those limits are true, and may be used in the present memoir which nowhere has occasion to explore the extremely small in space, or the extremely brief in time. 
The OEA, an Apparatus for making Observatory Experiments.
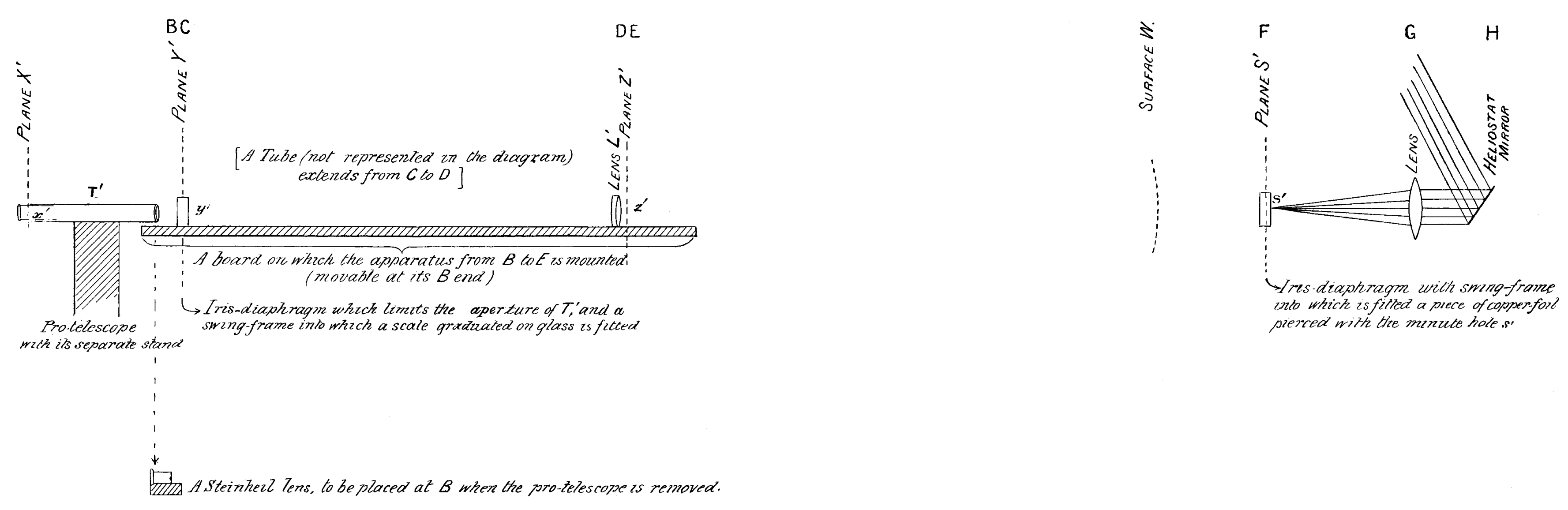

SCALE OF THE DIAGRAM, ONE-TENTH. 